\title{
La escritura de estudiantes extranjeros de la Universidad Nacional del Nordeste (UNNE). Fragmentariedad y distorsiones enunciativas
}

\author{
HUGO ROBERTO WINGEYER ${ }^{(1)}$ \\ MARÍA VIRGINIA BRUZZO(2)
}

Fecha de recepción 29/05/18

Fecha de aceptación: 01/09/18

Resumen. En el presente trabajo, enmarcado en el Proyecto de Investigación (PI 16H009) «Descripción y análisis de prácticas letradas académicas de estudiantes de la Facultad de Humanidades de la UNNE 2017/2020", nos proponemos revisar propuestas superadoras del enfoque comunicativo que sostiene que la lengua es un instrumento de comunicación, que sigue siendo utilizado actualmente en nuestra universidad tanto para enseñanza de L1 como para la de L2/E. En esta investigación, nos enfocamos en los estudios del español como L2/E y, más específicamente, en la expresión escrita. Para eso, analizamos un corpus de producciones escritas de estudiantes de intercambio de la UNNE, provenientes de Brasil y de República Checa que cursaron en estos últimos años la materia «Taller de Comprensión y Producción de Textos», en la

(1) Profesor en Letras. Magister en Enseñanza de Español como Lengua Extranjera y Doctor por la Universidad de Alcalá en el Programa de Lingüística Aplicada. Profesor Titular del Taller de Comprensión

\author{
y Producción de Textos en \\ la Universidad Nacional \\ del Nordeste. Director del \\ Instituto de Letras de la \\ Facultad de Humanidades de \\ la UNNE. Docente investigador, \\ director del PI I6HOO9 \\ «Descripción y análisis de
}

que introducimos a nuestros alumnos de español como lengua materna al discurso académico. Observamos, sobre la base de los conceptos de «fragmentariedad» y «distorsiones enunciativas» — propuestos por Desinano (2009) y García Negroni y Hall (2010) en el marco teórico de la adquisición de la lengua materna del interaccionismo brasileño, la polifonía argumentativa y los estudios discursivos - fenómenos que dan cuenta de las relaciones que el sujeto establece con la lengua, en el proceso de constituirse en sujetos del discurso académico (García Negroni y Hall, 2010).

Palabras clave. estudiante universitario de Español/Lengua Extranjera (E/LE) · expresión escrita - fragmentariedad $\cdot$ distorsiones enunciativas
Letras e integrante del grupo de investigación del PI I6HOO9 «Descripción y análisis de prácticas letradas académicas de estudiantes de la Facultad de Humanidades de la UnNE 2017/2020".

virginiabruzzo@gmail.com 


\section{Foreign students' writing at Universidad Nacional del Nordeste of Argentina (UNNE). Fragmentariness and enunciative distortions}

at UNNE, in which native Spanish speaking students are introduced to academic discourse. On the basis of the concepts of 'fragmentariness' and 'enunciative distortions' proposed by Desinano (2009) and García Negroni and Hall (2010) in the theoretical framework of Brazilian interactionism on language acquisition, argumentative polyphony, and discursive studies-, we observed phenomena that account for the subject's relationships with language in the process of becoming subject of academic discourse (García Negroni, 2010).

Keywords. SFL student at university - written expression $\cdot$ fragmentariness $\cdot$ enunciative distortions

\section{Introducción}

En el presente trabajo analizamos expresiones escritas de alumnos extranjeros de programas de intercambio con universidades latinoamericanas y europeas, con distinto nivel de conocimiento de español y diferente grado de adquisición del discurso académico; quienes, al mismo tiempo que tomaban clases de español como lengua extranjera, cursaban materias en la Facultad de Humanidades y en otras facultades de la UNNE.

Observamos que para acceder a programas de intercambio los estudiantes extranjeros deben cumplir en sus respectivas universidades con la aprobación de por lo menos el cuarenta por ciento (40\%) de sus planes de estudio, lo que hace suponer que llegan a nuestras aulas con cierto conocimiento del discurso 
académico. De todos modos, se les ofrece aquí asistir a la cátedra de primer año «Taller de comprensión y producción de textos» de la Facultad de Humanidades, en la que guiamos a nuestros alumnos ingresantes de las diferentes carreras en los primeros estudios de la comunicación de contenidos disciplinares.

En lo que respecta a nuestro análisis, tomamos en consideración seis muestras de un corpus de expresiones escritas de 10 estudiantes de intercambio de universidades de Brasil y de República Checa. Se trata de trabajos prácticos y exámenes parciales en los que se integran destrezas de comprensión lectora y expresión escrita, realizados en la mencionada materia de primer año.

Por último, aclaramos que, por un lado, las clases de E/LE que los estudiantes toman en la Facultad de Humanidades se hallan enmarcadas en el enfoque comunicativo. Mientras que, por el otro lado, en las clases de «Taller de Comprensión y Producción de Textos», que aunque también se enmarca básicamente en el enfoque comunicativo, se exploran constantemente otras alternativas superadoras de este enfoque, como la «fragmentariedad» (Desinano, 2009; García Negroni y Hall, 2010), las «distorsiones enunciativas» (García Negroni y Hall, 2010) y la "alfabetización semiótica» (Camblong y Froilán, 2012), teniendo presente siempre que aquello a lo que apuntamos es el desarrollo y la consolidación de la competencia académica.

\section{El tratamiento de la expresión escrita en el método comunicativo de E/LE}

Son varios los métodos de enseñanza-aprendizaje de E/LE, enmarcados en la Lingüística Aplicada. La expresión escrita ha tenido distinto tratamiento en los métodos más relevantes: el método tradicional o gramatical, el método de base estructural y el enfoque comunicativo.

En principio, en el método tradicional, se asocia la escritura con la adquisición de la gramática de la lengua meta, su objeto de enseñanza son las reglas gramaticales y las unidades léxicas del diccionario; y, por ende, se desarrollan en clase ejercicios de gramática y traducciones de textos literarios (Santos Gargallo, 1999). A su vez, en el enfoque estructural se concibe la escritura como un instrumento de refuerzo de la oralidad; se la emplea sólo para la práctica de 
diálogos orales; por eso, en la clase de gramática, en la que se destacan los ejercicios de dictado, redacciones, ejercicios de transformación de frases o de manipulación sintáctica y actividades de rellenado de huecos, las funciones son muy limitadas, con una concepción normativa alejada de lo dialectal, del contexto y de las situaciones, sin potenciar en ningún momento su dimensión creativa; en otros términos, el escrito es concebido como un producto, sin atender al proceso de escritura (Santos Gargallo, 1999).

Por último, el enfoque comunicativo, que se origina en los años 70 avalado por el Consejo Europeo, significó una renovación y superación de la metodología de la enseñanza de lenguas predominante por haber tomado aportes de la psicolingüística, la etnografía del habla, la pragmática, la sociolingüística, la lingüística, el análisis del discurso y el análisis de la conversación (Santos Gargallo, 1999). Considera la lengua como un instrumento de comunicación, con el objetivo del logro de la competencia comunicativa; logro que se alcanza en el desarrollo integrado de las cuatro destrezas lingüísticas: comprensión y expresión oral, comprensión y expresión escrita. En cuanto a esta última destreza, la expresión escrita, constituye una práctica compleja, si se tiene en cuenta que, a diferencia de la adquisición oral, «aprender a escribir requiere una instrucción formal que dura muchos años, que quizás no termina nunca» (Cassany, 2005:7). Se la trabaja, distinguiendo siempre necesariamente entre el proceso y el producto, casi siempre integrada a las otras destrezas. En la expresión escrita como producto se consideran la adecuación, la variación o estilo, la coherencia, la cohesión y la corrección gramatical. Respecto de la expresión escrita como proceso es posible distinguir pasos: la selección del tema; la preparación para la producción mediante actividades guiadas; la preparación de borradores por parte de los alumnos, revisados por el profesor y los propios alumnos; la preparación y redacción del producto final; y, finalmente, su corrección. En definitiva, consideramos que la expresión escrita desempeña un papel muy importante en la enseñanza comunicativa como una habilidad que tiene sus propias técnicas y objetivos.

En lo que respecta a los alumnos de intercambio, juntamente con nuestros estudiantes de L1, nuestro interés se centra en que adquieran las destrezas para producir textos expositivos (informes, monografías, artículos) que describen, explican y organizan nuestros conocimientos sobre el mundo, caracterizados 
por el predominio de la información, expuesta de manera ordenada, objetiva, clara y precisa. Se trata de textos en los que el lenguaje es empleado como instrumento, pero a la vez como medio de representación, interpretación y comprensión de la realidad (Montolío, 2014).

En general, esperamos que el alumno universitario adquiera los rasgos de los géneros académicos: «lenguaje preciso, riguroso y relativamente impersonal, una lógica argumentativa impecable, la máxima objetividad, y un propósito informativo explícito» (Reyes, 2009: 220). Sin embargo, muchas veces las dificultades en la producción escrita nos obligan a los profesores, en la lectura de los trabajos, a reponer la información faltante o reformular partes del texto para poder construir sentido. Es por esto que, a menudo, cuando corregimos reparamos solo en el nivel de los contenidos de los textos, relegando a segundo plano lo que García Negroni y Hall (2010) denominan «modos de decir». Esto significa que «damos por aprobadas evaluaciones (exámenes parciales, monografías, etc.), muchas veces porque hemos leído en ellas lo que los estudiantes «quisieron decir» pero sabemos que no dijeron» (García Negroni y Hall, 2010: 66).

\section{La búsqueda de enfoques superadores. Sobre la «fragmentariedad» $\mathrm{y}$ «las distorsiones enunciativas»}

Actualmente, nuestro equipo de investigación, que integra el PI 16H009 «Descripción y análisis de prácticas letradas académicas de estudiantes de la Facultad de Humanidades de la UNNE 2017/2020», busca propuestas en las que "la lengua no es concebida como un instrumento de comunicación que transmitiría sin más un contenido preexistente, ni los sujetos son considerados omnipotentes para realizar una supuesta comunicación» (García Negroni y Hall, 2010: 43), propuestas en las que la concepción de corrección gramatical sea reemplazada por otros criterios. Así adoptamos el concepto de «fragmentariedad», desarrollado por Desinano (2009), a partir del marco teórico de la adquisición del lenguaje encuadrado en el interaccionismo brasilero de Claudia de Lemos (1995, 2000). Según los estudios de Lemos (1995, 2000), cuando el infans, el niño, se enfrenta a la lengua puesta en la palabra del otro, pasa por un proceso en 
el que es capturado por ella para así constituirse como sujeto en el lenguaje. Sobre la base de estas investigaciones, Norma Desinano (2009) propone en su trabajo que la misma situación del niño ante la lengua se da en todos los momentos en los que el sujeto se enfrenta a un discurso desconocido, como es el caso de los alumnos universitarios a la hora de comprender y producir discursos académicos. En las producciones escritas de sus alumnos encuentra dificultades que, considera, "son el resultado de las interacciones entre el sujeto y la lengua que se plantean en el funcionamiento discursivo de ese sujeto particular» (Desinano, 2009: 91). Estos fenómenos lingüísticos-discursivos que dificultan de alguna manera la continuidad de los textos académicos conforman lo que Desinano (2009) denomina el "polo de la fragmentariedad».

Más tarde, García Negroni y Hall (2010), a partir de las ideas de Desinano (2009) y puestas en relación con la polifonía argumentativa (Bajtín, 1982 y Ducrot, 1984) y de los estudios discursivos (Arnoux et al., 2002 y Cassany, 2006), proponen los conceptos de «procedimientos de fragmentariedad» $y$ «distorsiones enunciativas». Los mismos son una sistematización de los fenómenos señalados por Desinano (2009), desarrollados con el propósito de «mostrar las relaciones que los estudiantes universitarios establecen con la lengua y, en particular, con el discurso académico» (García Negroni y Hall, 2010: 44). Según las autoras, las puntuaciones arbitrarias, las mayúsculas inesperadas, las abreviaturas personales, los blancos en el texto, los nexos que llevan a la ambigüedad y las relaciones anafóricas imprecisas son algunas de las marcas que conducen a la «fragmentariedad». Mientras que las distorsiones enunciativas son procedimientos discursivos en los que se manifiestan dificultades de redacción. Presentan las siguientes distorsiones, en el nivel dialógico-polifónico: distorsiones polifónicas; en el anclaje deíctico de los textos: distorsiones deícticas; y en el ámbito de las relaciones argumentativas: distorsiones argumentativas (García Negroni y Hall, 2010).

Por otra parte, dentro del PI, tomamos también en consideración los estudios sobre alfabetización semiótica de Ana Camblog y Froilán Fernández (2012), que retoman la línea teórica de Yuri Lotman (1996) con el concepto de «semiósfera» -si bien, no lo consideramos en particular en este trabajo, lo mencionamos brevemente por su pertinencia en el marco general de las investigaciones sobre las prácticas letradas académicas-. Estos autores proponen superar el 
reduccionismo del enfoque, antes mencionado, que considera que la lengua es «un instrumento de comunicación»; ya que:

1) el lenguaje supone una potencia semiótica que excede ampliamente lo instrumental; 2) concebir el lenguaje como instrumento supone que lo manejamos desde fuera, que operamos con el lenguaje desde un sujeto a priori, lo que implica excluir al lenguaje de la instauración y constitución misma de la conciencia semiótica; 3) la comunicación no agota las posibilidades semióticas del lenguaje. (2012: 59-60)

En cuanto a la situación de nuestros alumnos universitarios de ELE, quienes además de estar expuestos a la lengua española en la vida diaria con el complemento de clases formales en las que desarrollan la comprensión y la expresión oral, se enfrentan en las clases universitarias a la compresión y expresión escrita de discursos académicos. En su proceso de adquisición de la competencia académica, hemos encontrado en sus producciones con los mismos fenómenos que observamos en nuestros alumnos de L1: «procedimientos de fragmentariedad» $\mathrm{y}$ «distorsiones enunciativas». Es por ello que, siguiendo la hipótesis enunciada por Desinano, «la propuesta del interaccionismo respecto de las posiciones del sujeto en relación con la lengua durante la adquisición pueden considerarse como una constante del funcionamiento del sujeto en Lenguaje» (2009: 93), consideramos que nuestros estudiantes de intercambio se encuentran en la misma posición que el niño frente a la lengua materna o el estudiante universitario frente al discurso académico, sin desconocer la complejidad adicional de tener que desarrollar la competencia académica en una lengua extranjera. En resumen, si bien los estudiantes extranjeros que llegan a nuestras aulas, como ya se ha mencionamos, ya deberían haber tenido un importante desarrollo del discurso académico en sus lenguas maternas, según lo observado en muchos casos, la continuación del aprendizaje del español y la adquisición del discurso académico-científico se dan simultáneamente. 


\section{Análisis del corpus}

Tomamos seis muestras de un corpus de expresiones escritas de estudiantes extranjeros de la UNNE. Las tres primeras corresponden al análisis de la redacción de un resumen o síntesis de un texto de opinión: Un Mundo con Drogas de Jaime Baryilko; y, las tres últimas, a la redacción de un breve informe que integra las actividades de comprensión lectora de un artículo periodístico: Novios cama adentro.

\section{Muestras de resumen 0 síntesis}

Observamos, en principio, que la consigna de la actividad: "Haga un resumen o síntesis del texto en 4 o 5 párrafos», permitía que el estudiante optara por hacer una síntesis o un resumen, acorde con sus habilidades para el desarrollo de la escritura.

En el caso uno (Figura 1), que corresponde a una alumna de origen brasileño, observamos, ya en una primera mirada, que la consigna que debería ser un apoyo o guía, para el alumno, se presentó como una primera barrera, ya que como vemos, la alumna en vez de presentar los 4 o 5 párrafos requeridos, escribió sólo uno. Bien puede aplicarse esta cita de Desinano a esta dificultad:

[...] los alumnos no son orientados por la consigna a menos que ésta — como significantehaya formado parte de algún modo de una interacción previa en relación con el mismo discurso. En otras palabras, que el recorrido discursivo planteado por la consigna remita a un recorrido discursivo significable por el alumno. (2009: 47)

Además, aunque haya uso de varios marcadores discursivos-también y de igual manera-, la conexión de la tercera y cuarta oración con respecto al resto del texto es difusa debido al uso ineficiente de otros recursos lingüísticos que le hubieran dado como resultado un texto más cohesivo. En la tercera oración se introduce el relacionante cómo, sin embargo, no está presente la oración principal de la que dependería la subordinación introducida. Suponemos que 


\section{Preaente un resumen o sintesis del texto en 4 o 5 partes.}

Et teraa de las drogas se planien prineipalmente en la preganta: ipoe qué lo hacen? Con eso intentan encontrar una respuesta para las posibles causas y factores que isfluencian para que las personas hagan el consumo, muchas veces exugerajo, de drogas. Tumbietri, como la actual situación del mundo, fos problemas, las catástrofes ayuda a que uno vaya buscar en les itogas una posible "solación' para todto eso, o mejor; para no ver a todo eso. De igual manera, se plantea el hecho de que hay varias maneras de drogarse, como por ejemplo con juegos, internet, u otras couss que haga de la persona una depeadiente.

por el uso del conector también, la oración principal estaría en la anterior, aunque, como se ve, los recursos utilizados para conectar ambas ideas no fueron efectivos. Finalmente, en la cuarta oración, se menciona que también hay otros tipos de adicciones no relacionadas con el uso de sustancias psicotrópicas, sin marcar claramente la relación entre este dato con la información que nos proporciona el texto. Es por estas razones que pareciera que la alumna solo recogiera fragmentos de ideas del texto disparador, sin conseguir dar consistencia a su texto. Acorde con Desinano, podemos sostener que el sujeto aún no ha sido «capturado» o «alienado» por el discurso, entonces sólo recupera «fragmentos de textos otros y tanto la ausencia de elementos que los organicen como su empleo fallido, son las únicas marcas de subjetividad que demuestran la existencia de un discurso» (2009: 130). Afirmamos, entonces, que el discurso en el que la alumna aún no se ha constituido como sujeto es el del discurso académico, en primer lugar; y, el de la lengua española, en segundo lugar. Lo decimos porque, si bien presenta alguna dificultad a nivel gramatical —como por ejemplo, el uso de a ante OD -persona en no ver a todo eso-, evidencia un dominio aceptable de la lengua española; es, junto con las otras cuestiones planteadas, la elección entre resumen o síntesis la que representa una barrera para la alumna.

En el caso dos (Figura 2), otra estudiante brasilera, advertimos, desde lo visuográfico, que enumerar los párrafos, ya en una primera mirada, causa un efecto de «fragmentariedad», porque la sensación que se percibe es la de encontrarnos frente conjuntos de oraciones y no ante un texto. Asimismo, advertimos la falta de marcadores discursivos, que confirma la desconexión entre las oraciones. Igualmente, las dificultades con el español hacen que el 


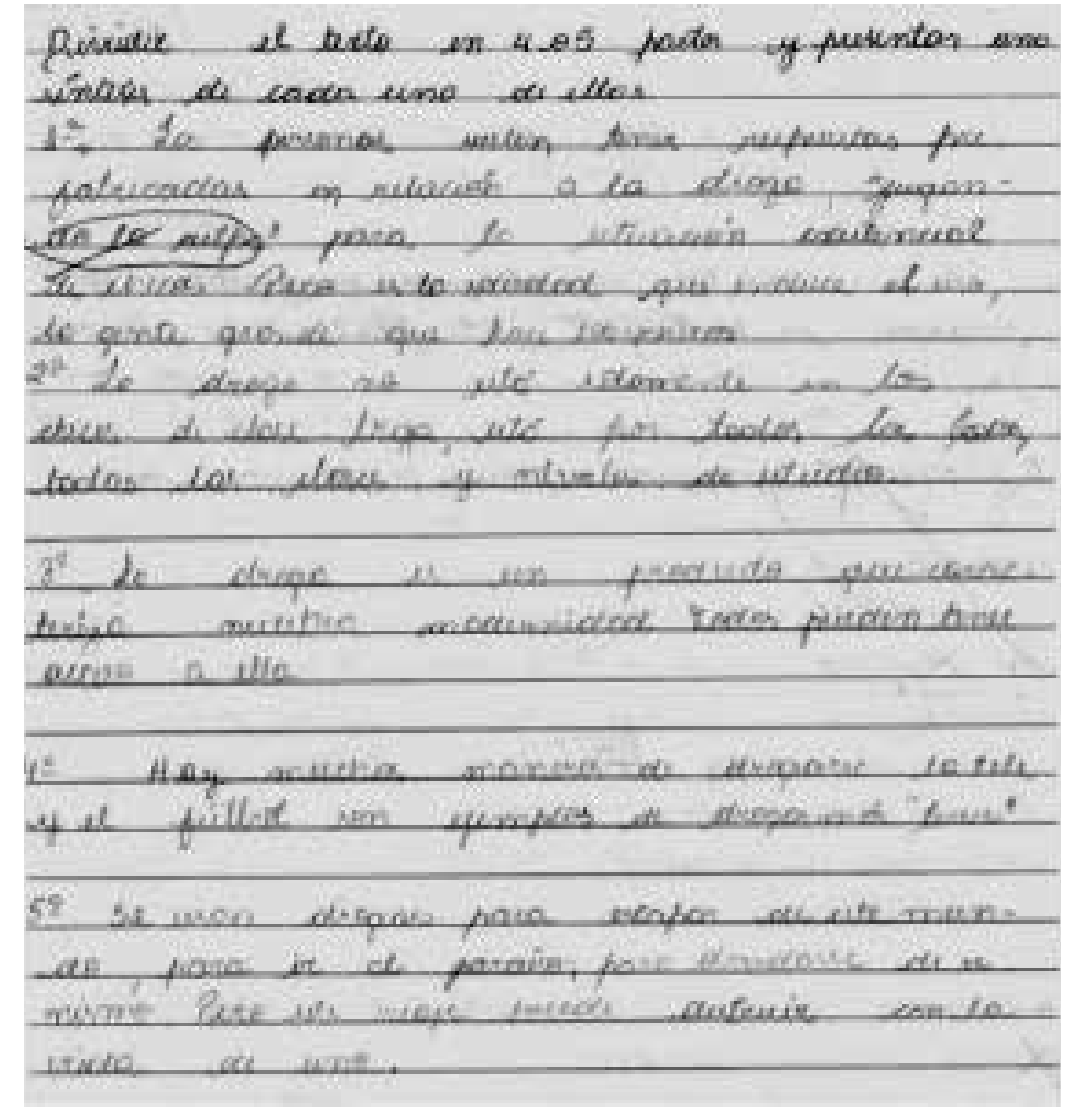

Figura 2

lector tenga que hacer un gran esfuerzo para dar sentido al texto, por ejemplo: «juzgando la culpa» para la situación existencial y la gente grande que hace los chicos. En lo que respecta a la competencia académica, podemos sostener que no reconoce el formato resumen o síntesis. En otras palabras, al igual que en el caso anterior, la consigna no es reconocible como texto posible (Desinano, 2009).

En el caso tres (Figura 3), una alumna de República Checa, advertimos que, en cierta medida, el sujeto ha sido "capturado» por el discurso académico 


\section{El resumen}

Les que consumen deogas si mismos muchas veces no saben explicar por gué. tn is mayoria de ias cases se puede tratar se varios persinales probiemas. La droei resresenta una salida que entonces al fin conduce obstacules mass grandes.

No es vertad somo dice o sasiene in general canocimiento sobre drogas. Las no se mocian solamerte a les chicos a pobres. La eipetiencia de personas de varias posiciones sociales habla por todos. Tambien no serie juste relaclonar drogas a este sigla. En el pasado pedriames encontrar muchos ejemplos y situaciones donde se habian vasde.

El mundo de hoy liena muchas angustias y preccupsciones. Vivimos en la sociecad material, ta de censume y rivalidad universal to que se sibraye en gran medida es cuarto. Menudo por el escape al munde fictico reempistames 10 que faltamos. Ista ensolación sin embargo produce decepción si nuestras ideas no corresponden con la reaidad.

Cada adicoión es el hecho problembtica. Desole adicoiones menos graves llegamos a tas más graves. Unas respuestas o razonamientos para explicar la base de este tema tenemos que buscaf en nuestra cabeza, mejor dicho, pricologla Es came el cínculo vicieso. No se podemes hartar sersaciones auradabies gracias a drogas pero dejar de uso es para nosatros inimagirable.

Figura 3

(Desinano, 2009). Por un lado, podemos distinguir los cuatro párrafos requeridos en la consigna; por otro, en una lectura rápida podemos observar que en cada párrafo se sostiene una unidad temática/semántica: en el primero, por qué se consumen drogas; en el segundo, los prejuicios sobre este consumo; en el tercero, la sociedad actual y su relación con el consumo de drogas; y, finalmente, en el cuarto, una conclusión.

De todos modos, el español representa una barrera para el sujeto ya que notamos varios rasgos en su «interlengua» que dan cuenta de la falta de dominio de la lengua, que son la causa del efecto de "fragmentariedad" que produce la lectura del texto, por ejemplo, en el uso de pronombres: Los que consumen drogas sí mismos, Las no se asocian; y en el orden de palabras, personales problemas por problemas personales. En síntesis, estamos en condiciones de sostener que, en este caso, el sujeto aún está en el proceso de «captura» de la lengua española pero, suponemos que, en 
su lengua materna o incluso en alguna otra lengua, ya ha sido «alienado» por el discurso académico.

\section{Muestras de informes}

Para esta actividad, la consigna dada fue: «Elabore un informe en el que exprese su punto de vista poniéndose en el lugar de un psicoanalista».

En el caso cuatro (Figura 4), alumna brasilera, notamos que la consigna, al igual que para los casos 1 y 2 , no es reconocible como texto posible (Desinano, 2009). En su argumentación, la estudiante no da su opinión de manera clara, sino que se limita a traer fragmentos, "cadenas latentes», de otros textos relacionados con la literatura antigua, la religiosidad y el casamiento, que no contribuyen en nada a la configuración de una opinión propia. Además, entre la amalgama de «fragmentos», encontramos una cita del texto disparador haciendo referencia a lo dicho por la psicoanalista Norma Barros en el que se elide la comilla de cierre; es decir que, «hay una falta de dominio de las prácticas discursivas académicas habituales para la introducción de voces ajenas en el propio discurso» (García Negroni y Hall, 2010: 54). Podemos sostener, en consecuencia, tomando la clasificación presentada por García Negroni y Hall (2010), que encontramos aquí un caso de distorsión argumentativa, no tanto por inconsistencias o incoherencias como es concebida en la bibliografía mencionada, sino por una falta de conexión entre los discursos traídos al texto y la falta de opinión propia. Finalmente, respecto del dominio de la lengua española, la alumna no presenta dificultades importantes en el orden de lo gramatical. A diferencia del caso 3, aquí el sujeto ya está en una posición más avanzada en el proceso de captura de la lengua española.

En el caso cinco (Figura 5), alumna brasilera, como primer rasgo de «fragmentariedad» notamos que el texto está escrito en tercera persona; es decir, que el sujeto no se posiciona como enunciador de su propio discurso, sino como enunciador de un discurso otro. Como refuerzo de este procedimiento de extrañamiento, se refiere a sí misma como un otro con la construcción 


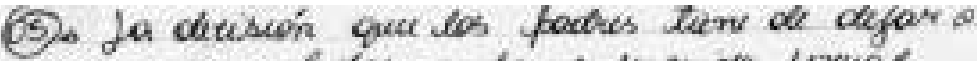

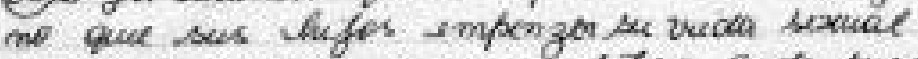

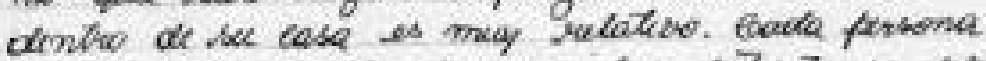

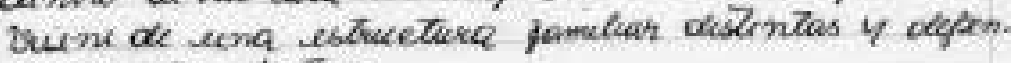
de de thes kaetores

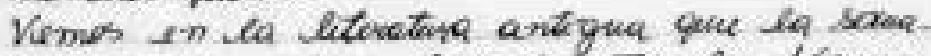

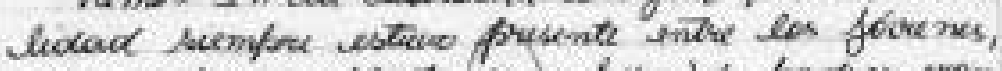

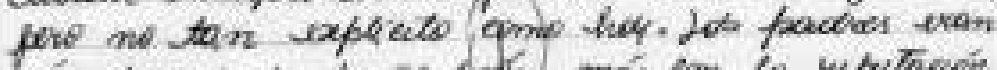

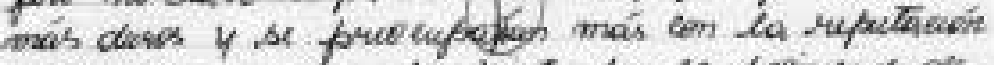

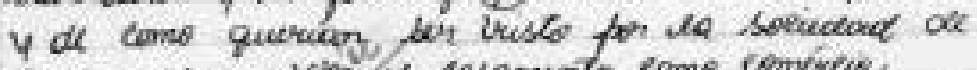
cacka epeca. vela le casamento come comvicie.

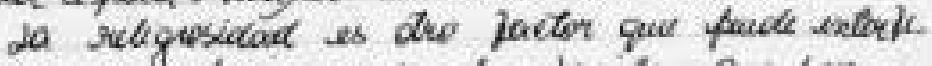
tet en las diescones. Ion las jamilias que son eristanas ne se parsition que ses higos lengar

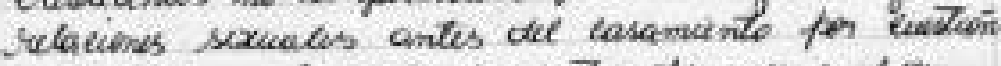
de respotio a los mendamicites dirumes y for segutwiad.

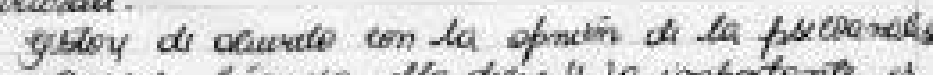

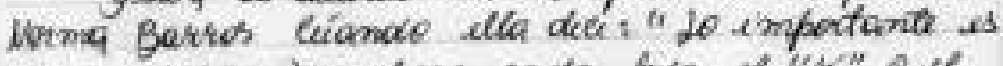
rewt qui signikea para eada bujo el "sc" o el "no" de los padtes

Figura 4

Para Aline... es importante que. Podríamos hipotetizar que el sujeto tiene en mente el texto disparador en el que para referir la opinión de una de las especialistas se utiliza la construcción Para la psicoanalista Norma Barros. Podríamos decir, en otras palabras, que la alumna trae un fragmento del discurso otro, el texto disparador, a su discurso a pesar de no ajustarse a lo requerido en la consigna. Además, notamos que la formulación de la opinión no es consistente puesto que, en primer lugar, la relación entre la primera oración es importante que haya un equilibrio en la presencia del novio (a) en el hogar y la segunda La permiso debe existir con reglas de permanencia no es clara; en segundo lugar, porque no se especifican cuáles serían las reglas de permanencia para que el hijo no se acomode con la situación. Finalmente, la elección del vocablo acomode no parece ser 


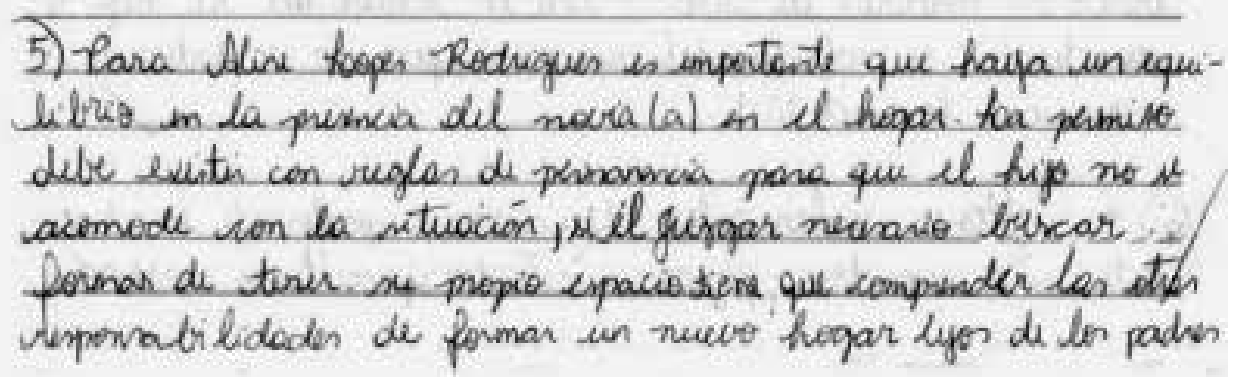

Figura 5

efectivo para la idea que intenta transmitir el sujeto que, suponemos, está en relación con la comodidad —vocablo fonéticamente similar al propuesto por la estudiante- . Podríamos decir que, al igual que en el caso anterior, nos encontramos ante una distorsión argumentativa. Finalmente, notamos algunas marcas que muestran el estadio de «interlengua» pero que no significan un impedimento mayor en la comprensión del texto, como el uso del artículo femenino la ante sustantivo masculino en La permiso; la conjugación en subjuntivo en si él juzgar en vez de si él juzgara y, finalmente, el infinitivo Terer en vez de Tener.

Finalmente, en el caso seis (Figura 6), alumna también brasilera, advertimos que, a diferencia de los casos anteriores, el sujeto se posiciona en el discurso, formula su opinión y la sostiene sin digresiones ni inconsistencias; es decir, cumple con el género discursivo requerido. Sólo registramos unos pocos fenómenos de nivel gramatical que dan cuenta de la «interlengua» del hablante, como el uso de la conjunción e ante $i$ como inicio de palabra - padres y hijos - y puntuaciones un tanto arbitrarias. Inclusive, notamos que el tachado de la preposición en reemplazada por a da cuenta de una «escucha» (Desinano, 2009) —posible cuando la relación sujeto/lengua/ discurso ya está establecida - que permitió a la alumna desandar sus pasos y corregir su propio discurso. Se podría decir, entonces, siguiendo la terminología propuesta por Desinano (2009), que se trata de un sujeto que ha logrado una «textualización» en el discurso académico en ELE. 


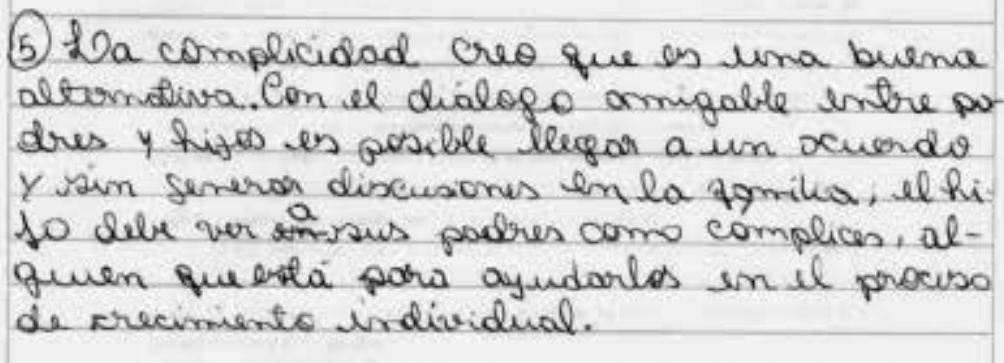

Figura 6

\section{Conclusión}

Como hemos observado en los casos analizados, los alumnos universitarios extranjeros, al igual que el «infans», para Lemos, y el alumno universitario en lengua materna, para Desinano, cuando llegan a nuestras aulas, se encuentran con un discurso puesto en la palabra del otro, y tienen que ser "capturados» por ella para funcionar como sujeto en el lenguaje. Sin embargo, a diferencia del «infans» o el alumno universitario en lengua materna, el extranjero pasa por un doble proceso de «captura», el de la lengua española y el del discurso académico-científico. Es por ello, que los conceptos de «fragmentariedad» $y$ «distorsiones enunciativas» nos resultan efectivos para la descripción de los fenómenos lingüísticos-discursivos producidos por los estudiantes.

En suma, podemos afirmar que cuando nos proponemos introducir a los alumnos extranjeros en el discurso académico en español como lengua extranjera, nos enfrentamos al desafío de acompañarlos en el proceso de constitución de sujetos de discurso. Proceso que necesitamos favorecer a través de la incorporación, tanto en las clases de E/LE, como en las clases de las materias que nos competen en la Facultad, de prácticas de lectura, escritura que se correspondan con este marco conceptual. 


\section{Referencias bibliográficas}

Arnoux, E., Di Stefano, M y Pereira, C. (2002). La lectura y la escritura en la universidad. Buenos Aires: Eudeba.

Bajtín, M. (1982). Estética de la creación verbal ( $5^{\circ}$ ed. Trad. T. Bubnova). México: Siglo XXI.

Camblong, A. y Fernández, F. (2012). Alfabetización semiótica en las fronteras. Volumen I. Dinámicas de las significaciones y el sentido. Posadas: EDUNAM. Editorial Universitaria de la Universidad Nacional de Misiones.

Cassany, D. (2004). La expresión escrita. En J. Sánchez Lobato e I. Santos Gargallo (Dir.), Vademécum para la formación de profesores. Enseñar español como segunda lengua (L2) / lengua extranjera (LE), pp. 917942. Madrid: Sociedad General Española de Librería. Cassany, D. (2005). Expresión escrita en L2/ELE. Madrid: Arco Libros.

Cassany, D. (2006a). Taller de textos. Leer, escribir y comentar en el aula. Barcelona: Paidós.

Cassany, D. (2006b). Análisis de una práctica letrada electrónica. Páginas de Guarda (2), 99-112.
Desinano, N. (2009). Los alumnos universitarios y la escritura académica. Análisis de un problema. Rosario: HomoSapiens.

Ducrot, 0. (1984). Le dire et le dit. París: Minuit.

García Negroni, Ma. M. y Hall, B. (2010). Escritura universitaria, fragmentariedad y distorsiones enunciativas propuestas de prácticas de lectura y escritura focalizadas en la materialidad lingüístico-discursiva. Boletín de Lingüística, 22 (34), 41-69.

Lemos, C. de (1995). Língua e discurso na teorização sobre aquisição de linguagem. En: Letras de Hoje, 102, pp. 9-29.

Lemos, C. de (2000). Questioning the notion of development: the case of language acquisition. En: Culture \& Psychology, 6, (2), pp. 169-182.

Lotman, Y. (1996). La semiosfera. Madrid: Cátedra. Montolío, E. (2014). Manual de Escritura Académica y Profesional (vol. 2). Barcelona: Ariel.

Reyes, G. (2009). Cómo escribir bien en español. Madrid: Arco Libros.

Santos Gargallo, I. (1999). Lingüística aplicada a la enseñanza-aprendizaje del español como lengua extranjera. Madrid: Arco Libros. 